\title{
Analyse vibratoire de modifications de structures. L'approche des cercles de Vincent revisitée
}

\author{
Olivier Tanneau ${ }^{1, a}$, Olivier Sauvage ${ }^{2}$ et Jean-Marie Lagache ${ }^{2}$ \\ 1 Société ALTRAN AIT, 2 rue Paul Vaillant Couturier, 92300 Levallois-Perret, France \\ 2 PSA Peugeot Citroën, VV 57, Route de Crisy, 78943 Vélizy-Villacoublay, France
}

Reçu le 31 janvier 2007, accepté le 7 février 2008

\begin{abstract}
Résumé - Nous traitons ici de la conception de systèmes anti-vibratoires venant modifier des structures complexes. L'approche développée utilise en entrée des modèles en éléments-finis condensés, ou bien des systèmes représentés sous une forme simplifiée à partir de modèles fonctionnels, ou bien encore de données expérimentales. Cette approche peut s'utiliser pour évaluer rapidement le potentiel de concepts et procéder à un premier dimensionnement, mais aussi pour dimensionner finement ces mêmes concepts au cours des cycles de conception. Nous utilisons comme point de départ les formules classiques de raccord impédanciel, que nous exploitons avec les propriétés homographiques identifiées par A.H. Vincent [1]. La méthode proposée permet notamment de prendre en compte des amortissements, de contrôler les effets de la modification sur l'ensemble de la bande de fréquences d'étude, ainsi que d'évaluer la sensibilité aux paramètres de conception. Nous donnons ici une application de cette méthode au dimensionnement d'un joint antivibratoire pour une culasse de moteur Diesel.
\end{abstract}

Mots clés : Cercles de Vincent / dynamique / vibration / amortissement

\begin{abstract}
Vibrations analysis of modified structures. The Vincent's circles approach revisited. This paper deals with the design of vibrations suppression systems for complex structures. The proposed method is well suited for inputs coming from finite element or simplified models or from test data. It can be used in rapid dimensioning, allowing fast evaluation of concepts, but also in different iterations in the design process, giving fine and efficient information. The basis of the present work is the standard formulation of dynamics responses of structures modified by a localized impedance, in the way of the A.H. Vincent [1]'s circles theory. The resulting approach allows notably the dimensioning of damping, the control of effects on the whole frequency range and one evaluation of sensitivity of dimensioned parameters. As an example, the study of a sealing device the function of which is to insulate the cylinder head from injectors of a diesel engine is detailed.
\end{abstract}

Key words: Vincent's circles / dynamics / vibrations / damping

\section{Introduction}

Le contrôle des vibrations sur des structures industrielles s'effectue souvent par l'adjonction de traitements localisés tels que des modifications de raideur, de masse, ou l'utilisation de résonateurs mécaniques. Or, la détermination des paramètres fonctionnels de ces traitements (masse, raideur, amortissement) peut s'avérer délicate pour de tels systèmes au comportement vibratoire souvent complexe, même en première approximation.

\footnotetext{
a Auteur pour correspondance :

olivier.tanneau@altran.com
}

La méthode proposée ici permet une estimation rapide et fiable des potentialités d'une telle modification sur une réponse vibratoire. Elle s'avère particulièrement bien adaptée aux phases d'avant-projet. Elle peut aussi s'utiliser ensuite dans les phases de réglage fin des solutions.

Elle est basée en premier lieu sur les formules de raccord impédanciel, permettant la mise à jour des réponses dynamiques d'une structure connue soumise à des modifications à partir de fonctions de transfert. C'est un résultat classique (voir par exemple [2]) dont il existe de nombreuses applications [3-6], couvrant des problématiques aussi variées que l'optimisation de structure (dimensionnement), le diagnostic de structure en service ou le recalage de modèles. 


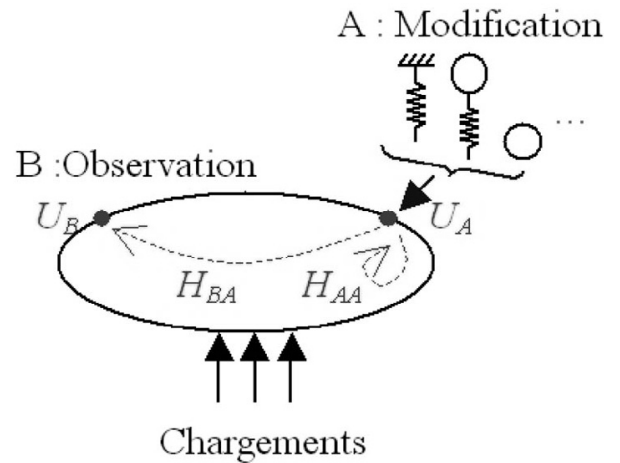

Fig. 1. Problème générique.

Elle se base dans un second temps sur les principes (moins connus) identifiés par Vincent [1] au début des années soixante-dix. Ce dernier remarque en effet que, pour une fréquence donnée, les réponses mesurées en service pour la structure à traiter se situent invariablement sur un cercle du plan complexe - pour des modifications simples telles que l'ajout de raideurs locales ou d'étouffeurs dynamiques. Vincent suggère alors l'utilisation de cette propriété pour l'optimisation de paramètres structuraux, problème alors difficile compte-tenu des capacités de calcul. Quelques publications relaient cette préoccupation en élargissant la technique à l'étude simultanée de plusieurs modifications structurales [7] ou encore dans l'évaluation de critères basés sur cette technique et liés à des modélisations par éléments-finis [8].

Pendant les deux décennies suivantes, cette méthode progresse peu et seuls quelques spécialistes des vibrations semblent s'en servir dans l'industrie, en particulier dans le groupe PSA Peugeot Citroën avec les travaux de Lagache [9]. Plus récemment, une publication [10] généralise l'approche au cas de plusieurs modifications en se concentrant sur l'étude du domaine des réponses possibles (cercle pour une simple modification, surface fermée dans le cas général), toujours dans le plan complexe pour une fréquence donnée.

Les approches présentées ici ont plusieurs originalités. Premièrement, elles permettent une résolution analytique des problèmes directs et inverses : «Quel est l'effet d'une modification? $»$ et «Quelle modification pour tel effet? $»$. Ensuite, elles autorisent la résolution de ces questions au sens d'intervalles tant pour les paramètres de modification que pour les réponses vibratoires, ce qui se traduit par un accès analytique aux études de potentialités ou de sensibilités. Enfin, elles permettent la prise en compte d'amortissements dans les modifications envisagées.

L'exposé présente tout d'abord les principaux fondements de la technique des cercles de Vincent, puis comment celle-ci a été utilisée pour la constitution d'outils d'optimisation de renforts élastiques amortis. Dans une seconde partie, une application est donnée dans le cas du dimensionnement d'un joint anti-vibratoire pour une culasse de moteur Diesel.

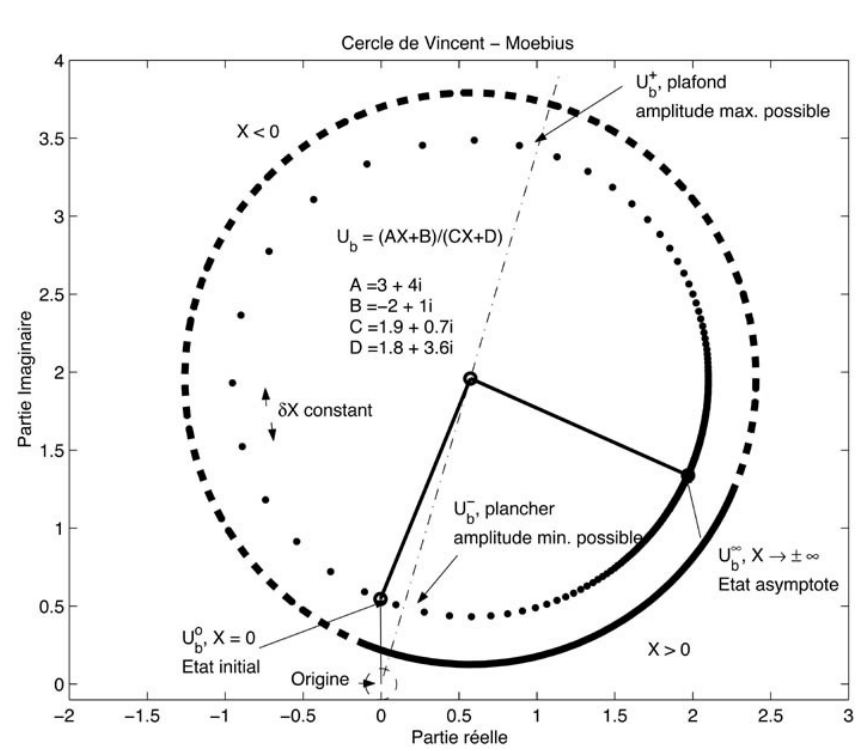

Fig. 2. Exemple de cercle de raccordement, lieu dans le plan complexe de la réponse $U_{b}$ possible en $B$ pour toute modification d'impédance $X=Z$ locale en $A$ (pour une fréquence donnée). Quatre points remarquables sont à considérer : (i) l'état initial $(X=0)$, (ii) l'état asymptote $(X \rightarrow \pm \infty$, les deux infinis se rejoignent), (iii) le point d'amplitude minimum (première intersection droite origine-centre du cercle au cercle) et (iv) le point d'amplitude maximum (l'autre intersection). Il est également important de séparer les arcs de cercle en fonction du signe de $\mathrm{X}$ (trait plein : $X>0$, trait en pointillés : $X<0)$.

\section{Bases théoriques}

\subsection{Principe général}

Le problème traité est celui représenté figure 1. Le point $A$ désigne la zone de modification à laquelle on associe le degré de liberté de couplage avec le renfort : indifféremment, une translation, une rotation, ou une combinaison de degrés de liberté en $A$. Le point $B$ désigne quant à lui la zone d'observation des vibrations. Elle peut éventuellement être confondue avec le point $A$. On note $U_{a}^{0}$ et $U_{b}^{0}$ les réponses en ces degrés de liberté de la structure initiale soumise aux chargements.

En supposant que la structure non sollicitée par les chargements reçoive une force $F_{A}^{1}$ au point $A$, les réponses obtenues en $A$ et $B$ peuvent s'écrire :

$$
\begin{aligned}
U_{a}^{a} & =H_{a a}^{0} F_{A}^{1} \\
U_{b}^{a} & =H_{b a}^{0} F_{A}^{1}
\end{aligned}
$$

Les transferts $H_{a a}$ et $H_{b a}$ sont respectivement la souplesse d'entrée en $A$ (déplacement en $A$ pour une force en $A$ ) et la souplesse de transfert de $A$ vers $B$. Les réponses totales en service $U_{a}^{Z}$ et $U_{b}^{Z}$ tenant compte de $F_{A}^{1}$ s'obtiennent directement par superposition :

$$
\begin{aligned}
& U_{a}^{Z 1}=U_{a}^{0}+U_{a}^{a}=U_{a}^{0}+H_{a a}^{0} F_{A}^{1} \\
& U_{b}^{Z 1}=U_{b}^{0}+U_{b}^{a}=U_{b}^{0}+H_{b a}^{0} F_{A}^{1}
\end{aligned}
$$


Nous introduisons le renfort (ou encore : la modification, la partie raccordée, le raccordement, ...) par une impédance $Z$ a priori complexe et variable en fréquence et rapportée au degré de liberté de couplage en $A$ tel que :

$$
U_{a}^{Z 2}=\frac{1}{Z} F_{A}^{2}
$$

$U_{a}^{Z 2}$ et $F_{A}^{2}$ correspondent à la réponse et à la force en $A$ vues du renfort. L'introduction des relations de couplage entre la structure et le renfort en $A$ :

$$
\begin{aligned}
& F_{A}^{1}+F_{A}^{2}=0 \\
& U_{a}^{Z 1}=U_{a}^{Z 2}
\end{aligned}
$$

permet d'assembler les relations (2) et (3) de façon à exprimer simplement la réponse de la structure $U_{b}^{Z}$ avec une modification locale $Z$ au point d'observation $B$ en fonction des réponses initiales en $A$ et $B$ et des souplesses initiales de la structure :

$$
U_{b}^{Z}=U_{b}^{0}-\frac{H_{b a}^{0} U_{a}^{0}}{H_{a a}^{0}+\frac{1}{Z}}
$$

Cette expression usuelle (analogue à l'Éq. (6.42) p. 486 de [2]) présente évidemment l'intérêt de permettre un calcul rapide de l'effet de modifications simples. Elle peut se généraliser à des renforts se connectant sur plusieurs degrés de liberté.

Pour un renfort agissant sur un ou deux degrés de liberté, l'équation (5) présente des propriétés analytiques remarquables généralement non présentées. Elle peut en effet se classer parmi les fonctions homographiques de la variable complexe $Z$ (ou transformation de Moebius [11]) :

$$
U_{b}^{Z}=\frac{A Z+B}{C Z+D}
$$

Si $Z$ est réel, $U_{b}^{Z}$ décrit un cercle (voir Fig. 2) et donc les extremums pour $U_{b}^{Z}$ peuvent facilement se calculer, de même que l'estimation de $Z$ pour un gain voulu. Dans le cas général où $Z$ est complexe, une séparation de variables, par exemple l'examen de la partie réelle ou imaginaire de $Z$, permettra toujours de se ramener à une expression à variable réelle $X$ (telle que 6 ). Un report des termes non utilisés est ensuite à effectuer vers les coefficients $A, B, C$ et $D$.

\subsection{Déclinaisons}

L'impédance $Z$ peut correspondre à de nombreuses familles de modifications. La formule (5) s'applique directement à l'ajout d'une raideur reliée à une base fixe $(Z=K)$, d'une masse $\left(Z=-\omega^{2} M\right)$, d'un résonateur $\left(Z=\left(\frac{1}{K}-\frac{1}{\omega^{2} M}\right)^{-1}\right)$, et de manière générale à tout renfort à impédance réelle agissant sur un seul degré de liberté.

Dans cet article, nous nous intéressons plus précisément à l'ajout d'une raideur parallèle entre deux points $A_{1}$ et $A_{2}$. Ce cas particulier nécessite les recombinaisons des données d'entrée suivantes :

$$
\begin{aligned}
& H_{a a}^{0}=H_{a 11}+H_{a 22}-H_{a 12}-H_{a 21} \\
& H_{b a}^{0}=H_{b a 2}-H_{b a 1} \\
& U_{a}^{0}=U_{a 2}-U_{a 1}
\end{aligned}
$$

avec, de façon à retrouver une formule du type de l'équation (5):

- $H_{\text {aii }}$ la matrice $2 \times 2$ de souplesse entre les deux degrés de liberté de connexion (points $A_{1}$ et $A_{2}$ );

- $H_{b a i}$ les deux transferts entre le point d'observation $B$ et les points $A_{1}$ et $A_{2}$;

- $U_{a 1}$ les réponses initiales en $A_{1}$ et $A_{2}$.

L'impédance $Z$ correspond dans ce cas directement à la raideur du ressort que l'on souhaite dimensionner. Il est également intéressant de vouloir associer un amortissement à ce ressort. Pour cela, nous prenons l'hypothèse d'un amortissement hystérétique tel que $Z=K(1+j \eta)$.

Les cercles de raccordement sont définis pour un paramètre appartenant à l'ensemble des nombres réels. Nous devons donc faire un choix entre l'étude de la raideur (à amortissement fixé) ou de l'amortissement (à raideur fixée). Dans le premier cas, la forme homographique est définie par :

$$
\begin{aligned}
& U_{b}(K)=\frac{A K+B}{C K+D} \\
& A= U_{b}^{0} C-H_{b a}^{0} U_{a}^{0}(1+j \eta) \\
& B=U_{b}^{0} \\
& C=H_{a a}^{0}(1+j \eta) \\
& D=1
\end{aligned}
$$

et dans le second cas par :

$$
\begin{gathered}
U_{b}(\eta)=\frac{A \eta+B}{C \eta+D} \\
A=U_{b}^{0} C-j K H_{b a}^{0} U_{a}^{0} \\
B=U_{b}^{0} D-K H_{b a}^{0} U_{a}^{0} \\
C=j K H_{a a}^{0} \\
D=K H_{a a}^{0}+1
\end{gathered}
$$

Ces deux problèmes sont complémentaires et une utilisation successive de chacun est implicitement suggérée : étude de la raideur, puis de l'amortissement par exemple. Pour faciliter les allers-retours, ces deux problèmes peuvent être modifiés de façon à faire apparaître une valeur initiale des deux paramètres du renfort et, comme paramètre $X$, une variation de raideur ou d'amortissement. Les formules s'écrivent alors, pour une variation de raideur de $\delta K$ :

$$
\begin{aligned}
& U_{b}^{\left[K_{0}, \eta_{0}\right]}(\delta K)=\frac{A \delta K+B}{C \delta K+D} \\
& A=U_{b}^{0} C-H_{b a}^{0} U_{a}^{0}\left(1+j \eta_{0}\right) \\
& B=U_{b}^{0} D \\
& C=H_{a a}^{0}\left(1+j \eta_{0}\right) \\
& D=1+K_{0} C
\end{aligned}
$$


et, pour une variation d'amortissement de $\delta \eta$ :

$$
\begin{gathered}
U_{b}^{\left[K_{0}, \eta_{0}\right]}(\delta \eta)=\frac{A \delta \eta+B}{C \delta \eta+D} \\
A=U_{b}^{0} C-j K_{0} H_{b a}^{0} U_{a}^{0} \\
B=U_{b}^{0} D-K_{0} H_{b a}^{0} U_{a}^{0} \\
C=j K_{0} H_{a a}^{0} \\
D=K_{0} H_{a a}^{0}+1+C \eta_{0}
\end{gathered}
$$

Ces différents cas ne sont que des exemples du très grand nombre de paramétrages possibles. Des renforts ou traitements de natures complètement différentes peuvent a priori être envisagés dès lors qu'une relation de type homographique du paramètre à dimensionner est établie.

\subsection{Critères et indicateurs}

\subsubsection{Objectifs}

Si la propriété mise en évidence par Vincent est connue depuis longtemps, elle a été relativement peu utilisée. En pratique, le tracé seul des cercles de Vincent s'avère décevant et limité. D'une part, un cercle très différent peut être obtenu pour chaque fréquence, et, d'autre part, les cercles sont parcourus avec une vitesse non constante, comme le laisse apparaître le tracé de la figure 2. Ainsi, selon les situations, certaines parties des cercles sont difficiles à atteindre avec des valeurs réalistes des paramètres.

Hanson et Calapodas [8] ont proposé l'établissement d'un critère de sensibilité basé sur le diamètre du cercle de Vincent. Cette technique, utilisée avec des modèles éléments-finis, a été évaluée comparativement à des critères classiques d'énergie vibratoire. Elle possède une bonne capacité à identifier les meilleurs emplacements pour une modification locale mais s'est avérée peu performante pour l'identification de zones d'action étendues.

De nombreux travaux internes au groupe PSA ont progressivement mis en avant la nécessité de fournir des informations et critères représentés en fonction de la fréquence et comportant des informations de sensibilité ou de potentialité. Cette expérience a été capitalisée et enrichie de développements théoriques et méthodologiques importants [9] sur lesquels s'appuient ces travaux.

\subsubsection{Courbes enveloppes}

Le cercle de Vincent est entièrement parcouru pour $X \in \mathbf{R}$. Si le cercle ne passe pas par l'origine du plan complexe, nous pouvons aisément constater que l'amplitude de la réponse modifiée ${ }^{1}$ est bornée par les amplitudes de deux états que l'on peut géométriquement déterminer par l'intersection du cercle et de la droite passant par l'origine et le centre du cercle (voir Fig. 2).

Le centre $O$ et le rayon $R$ du cercle se calculent par :

$$
\begin{aligned}
& O=U_{b}^{0}\left(1+\eta_{\text {car }}\right) \\
& R=\left|U_{b}^{0}\right|\left|\eta_{\text {car }}\right|
\end{aligned}
$$

\footnotetext{
1 C'est-à-dire la distance de l'origine au point initial.
}

où $\eta_{\text {car }}$ est une fonction caractéristique introduite par Lagache [9] dont l'expression vaut :

$$
\eta_{\mathrm{car}}=\frac{-j}{2 \operatorname{Im}\left(\frac{C}{D}\right)}\left(\frac{A}{B}-\frac{C}{D}\right)
$$

Les formules pour le calcul des extremums se résument alors à :

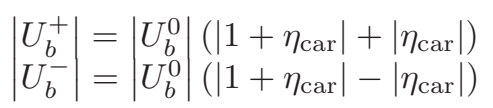

Le tracé en fréquence de ces deux courbes définit une enveloppe dans laquelle sont contenues toutes les réponses possibles pour l'ensemble des valeurs de $X$. Cette représentation permet donc un diagnostic rapide sur les potentialités absolues d'une modification indépendamment de la valeur et de la nature du paramètre $X$.

Il est préférable, en particulier pour les renforts parallèles, de réduire le champ des possibles pour $X$ à un intervalle borné. En effet, si $X$ représente une raideur, on s'intéressera plutôt aux valeurs positives ${ }^{2}$. De même, quand $X$ est un facteur d'amortissement hystérétique, la plage d'intérêt est souvent limitée : entre 0 et 0,5 par exemple.

Cette prise en compte ne pose pas réellement de problème. Il convient de bien identifier la position de l'arc de cercle correspondant à l'intervalle de $X \in\left[X_{1}, X_{2}\right]$ choisi. Si les extremums $U_{b}^{-}$ou $U_{b}^{+}$pour $X \in \mathbf{R}$ appartiennent à cet arc, ils sont les bornes recherchées; sinon il s'agit des limites $U_{b}^{X 1}$ ou $U_{b}^{X 2}$. Ces enveloppes plus resserrées permettent d'estimer les potentialités réalistes des solutions, avec des valeurs de paramètres respectant la physique ou les contraintes du problème.

La limitation à un intervalle choisi permet également l'évaluation d'enveloppes pour une petite variation d'une solution déjà identifiée : par exemple, la sensibilité pour un ressort $K$ avec une incertitude sur $K$ de $\pm 5 \%$. On peut ainsi, lors d'une phase de dimensionnement, investiguer la robustesse d'une solution.

\subsubsection{Problème inverse et dimensionnement}

Le caractère analytique de la fonction (6) autorise une résolution relativement aisée du problème inverse : l'estimation de $X$ pour une réponse voulue. Si l'amplitude de $U_{b}^{X}$ est fixée, selon que le cercle représentant ce niveau dans le plan complexe coupe, tangente ou passe à l'extérieur du cercle de Vincent, deux, une ou aucune solution $X$ peuvent être identifiées.

En partant du module de l'expression (6) et considérant $\left|U_{b}^{X}\right|$ imposé, il vient :

$$
X=\frac{-\operatorname{Re}\left(C D^{*}\right)\left|U_{b}^{X}\right|^{2}+\operatorname{Re}\left(A B^{*}\right) \pm \sqrt{\Delta}}{\left|U_{b}^{X}\right|^{2}|C|^{2}-|A|^{2}}
$$

\footnotetext{
${ }^{2}$ Les valeurs négatives peuvent correspondre à un assouplissement local, ce qui est parfois envisagé.
} 
avec :

$$
\begin{aligned}
\Delta=\left[\operatorname{Re}\left(C D^{*}\right)\right. & \left.\left|U_{b}^{X}\right|^{2}-\operatorname{Re}\left(A B^{*}\right)\right]^{2} \\
& -\left[|C|^{2}\left|U_{b}^{X}\right|^{2}-|A|^{2}\right]\left[|D|^{2}\left|U_{b}^{X}\right|^{2}-|B|^{2}\right]
\end{aligned}
$$

Les problèmes de dimensionnement posés en pratique ne sont jamais aussi simples que la détermination de $X$ garantissant une atténuation donnée à une fréquence donnée. Ils sont le plus souvent formulés en fréquence et plutôt sous la forme d'inégalités : une atténuation minimum par plages de fréquence par exemple. Aussi, nous proposons l'établissement d'une cartographie des gains pour la réponse $U_{b}^{X}$ dans un plan (fréquence, paramètre $X$ ).

Cette représentation s'obtient très rapidement par tracé des lignes d'isovaleurs de gains, que l'on obtient directement de la formule (19).

Une fois ce diagramme constitué, le dimensionnement proprement dit s'effectue par le report des objectifs d'atténuation dans le plan fréquence-paramètre, ce qui détermine des zones de passage pour le renfort. Si ces zones existent, il suffit alors de lire les valeurs de $X$ correspondantes et de faire un choix. Par exemple, sur la figure 5 présentée dans la partie 2 , le paramètre est une raideur. En supposant cette dernière constante en fréquence, le problème revient à positionner au mieux une droite horizontale dans ces zones. Dans l'exemple, il est ainsi possible d'atténuer la réponse initiale d'environ $10 \mathrm{~dB}$ sur les résonances entre $4 \mathrm{kHz}$ et $6 \mathrm{kHz}$ avec des valeurs acceptables de raideur.

Cet outil graphique présente l'intérêt de pouvoir traiter de nombreux problèmes d'optimisation sans changer de diagramme. Celui-ci est en effet indépendant des objectifs et des hypothèses sur les paramètres $X$ autres que son appartenance à l'intervalle considéré. Il est en particulier possible d'identifier sur ces représentations l'influence d'une dépendance en fréquence ou d'une incertitude sur la valeur de $X$

En traçant sur le diagramme les zones de passage (pour obtenir les gains désirés) et les zones à éviter (pour empêcher l'émergence marquée de nouvelles résonances), nous constituons un gabarit dans lequel le paramètre $X$ doit se situer. Le dispositif étudié ou envisagé, paramétré par $X$, peut être de nature physique plus ou moins complexe et dépendant de la fréquence : simple raideur, amortissement, système résonant, semi-actif, etc. Ce dernier peut alors être choisi puis dimensionné sur la base d'une spécification constituée à partir de tels gabarits.

\section{Exemple : traitement du bruit d'injecteur}

Nous développons ici un des multiples exemples d'applications possibles des méthodes proposées précédemment. Le cas choisi est celui du filtrage du bruit généré par les injecteurs des moteurs Diesel.

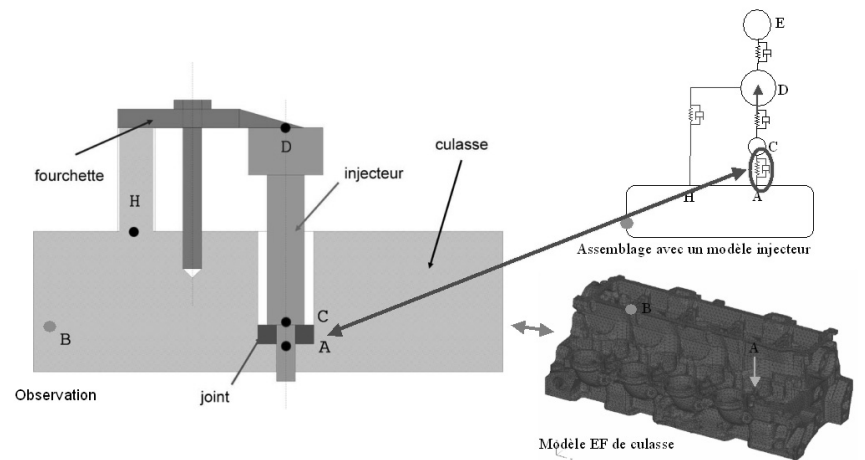

Fig. 3. Schéma d'un injecteur haute pression fixé sur la culasse d'un moteur Diesel, comprenant un joint anti-vibratoire positionné au niveau du nez de l'injecteur.

\subsection{Problématique}

Les systèmes et stratégies de combustion qui ont permis le succès des moteurs Diesel de nouvelle génération, comme la gamme HDi de PSA, utilisent des injecteurs de haute technologie. En contrepartie de l'efficacité pour le contrôle moteur et la combustion, les actuateurs utilisés dans les injecteurs haute pression sont une source de bruits et vibrations néfastes. Ces actuateurs peuvent être des solénoïdes ou, technologie plus récente, des piles piézoélectriques.

Le bruit généré par les injecteurs est impulsif et s'étend sur plusieurs milliers de Hertz, dans un domaine « moyennes fréquences » du point de vue de la longueur d'onde des vibrations et de sa situation dans le spectre $\mathrm{du}$ bruit moteur total. Une des solutions à ce problème est d'ajouter un joint anti-vibratoire [12] entre ceux-ci et la culasse où ils sont attachés, comme représenté sur le schéma de la figure 3.

Même sur un système a priori simple comme un joint filtrant, étudier sa faisabilité, donner rapidement un premier dimensionnement pertinent et effectuer un dimensionnement final précis et justifié ne sont pas des tâches triviales. Une raison est qu'il faut s'intéresser aux vibrations d'ensemble d'un système couplé et complexe.

L'application de la méthode présentée précédemment va nous permettre ici de pré-dimensionner au mieux le joint, c'est-à-dire choisir sa raideur et son coefficient d'amortissement hystérétique optimums. On se place ainsi dans les premières phases de conception et on spécifie les caractéristiques fonctionnelles de la solution technique.

Deux étapes pratiques sont à réaliser : d'une part la construction d'un modèle physique représentant l'ensemble culasse + injecteur, d'autre part la mise en œuvre des méthodes de raccordement dynamique sur ce modèle.

\subsection{Modèles utilisés pour représenter l'ensemble culasse et injecteur}

Une première étape est de choisir la représentation du système physique sur lequel on va raccorder une impédance : points d'entrée d'effort, points d'observation, 
point où se situe le renfort, transferts et réponses vibratoires entre ces points. Les transferts et réponses peuvent s'obtenir par calcul ou par essais : des approches mixtes peuvent évidemment être envisagées.

Pour l'application présentée ici, nous avons choisi les points suivants pour les entrées de la méthode de raccordement (cf. Fig. 3) :

- point D : point d'application de l'effort généré par l'injecteur ;

- point B : point d'observation des vibrations, placé sur la culasse;

- point $\mathrm{C}$ : nez de l'injecteur;

- point A : fond du puits accueillant l'injecteur au fond de la culasse;

- point H : point d'attache de la fourchette d'injecteur sur la culasse.

La culasse a été représentée par un modèle détaillé en éléments-finis (environ 200000 degrés de liberté) recalé sur ses premiers modes propres (une quinzaine entre 1 et $4 \mathrm{kHz}$ en conditions libres). Le comportement vibratoire de l'injecteur est représenté par un modèle masses-ressort à deux degrés de liberté et sa fixation par une raideur. L'injecteur est relié aux nœuds des surfaces de liaison de la culasse (voisinage des points $\mathrm{A}$ et $\mathrm{H}$ ) par des éléments de liaisons rigides. Les paramètres du modèle masses-ressort ont été identifiés par des essais sur l'injecteur seul. On attribue à l'ensemble un amortissement hystérétique uniforme de $1 \%$. Dans la suite on s'intéressera à des réponses vibratoires observées au point $B$.

Un point représentatif de l'utilité générale des méthodes de raccordement présentées ici est que la raideur de la fourchette a été déterminée en utilisant les développements méthodologiques précédents. En effet, connaissant expérimentalement les transferts et réponses en service sur l'ensemble initial culasse, injecteur, fourchette, on peut recaler rapidement la raideur de cette dernière en la considérant comme une impédance de raccord.

Une fois ces transferts connus par le biais de modèles représentatifs des sous-systèmes en jeu - qui comprennent leurs propres hypothèses de modélisation, nous appliquons la méthode de raccordement pour dimensionner les caractéristiques du joint découplant et amortissant.

\subsection{Utilisation de la méthode de raccordement dynamique}

Nous procédons maintenant au dimensionnement du joint anti-vibratoire placé entre le nez de l'injecteur (point C) et le fond du puits de la culasse (point A). Dans un premier temps nous allons utiliser comme paramètre de raccordement la raideur du joint, puis dans un second temps son coefficient d'amortissement.

\subsubsection{Paramètre de raideur}

Dans le modèle initial de l'ensemble culasse, injecteur, fourchette que nous avons choisi il n'existe pas de raideur

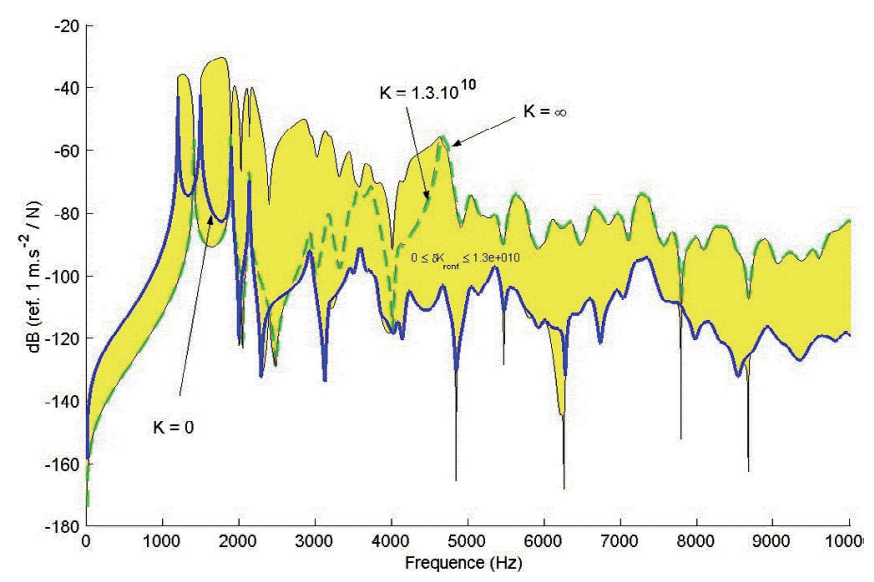

Fig. 4. Courbes enveloppes en fonction de la fréquence pour une plage de variations de la raideur. Zone grisée : enveloppe pour l'introduction d'un joint de raideur comprise entre 0 et $1,3 \times 10^{10} \mathrm{~N} . \mathrm{m}^{-1}$. Les pointillés correspondent au cas $K=$ $1,3 \times 10^{10} \mathrm{~N} \cdot \mathrm{m}^{-1}$ (joint cuivre) et la courbe en trait épais à l'absence du joint $\left(K=0 \mathrm{~N} \cdot \mathrm{m}^{-1}\right)$. Remarque : aucune de ces deux dernières courbes ne correspond totalement aux limites de l'enveloppe.

directe placée entre les points A et $\mathrm{C}$ (c'est cette raideur qui fait l'objet du raccord ajouté) : physiquement l'injecteur est donc découplé de la culasse à cet endroit. En réalité il existe un joint en cuivre de raideur élevée entre ces deux points et le cas correspondant à la situation réelle que l'on souhaite améliorer correspond à $K=1,3 \times 10^{10}$ N.m ${ }^{-1}$.

Le pré-dimensionnement va permettre de déterminer une valeur plus faible de cette raideur $K$ permettant un meilleur découplage.

Les calculs ont ainsi été effectués avec la plage de variation $\left[0-1,3 \times 10^{10}\right] \mathrm{N} . \mathrm{m}^{-1}$.

La figure 4 présente l'enveloppe pour cette plage de variation de $K$ (zone grisée), les cas correspondant à

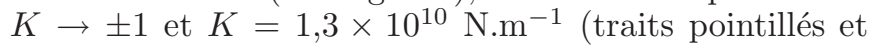
confondus) et le cas correspondant à $K=0$ (trait épais continu). Il apparaît ainsi, du point de vue vibratoire, que la raideur élevée due au joint cuivre donne un résultat quasiment identique à celui produit par une raideur infinie et que l'effet de couplage est très significatif à partir de $3000 \mathrm{~Hz}$. À partir du pic de résonance que nous souhaitons traiter, à $4600 \mathrm{~Hz}$, le cas correspondant au joint cuivre est superposé à la courbe enveloppe maximum et un potentiel de réduction de 15 à $20 \mathrm{~dB}$ peut être espéré. En dessous de cette fréquence de résonance, la tendance s'inverse : les niveaux se rapprochent du minimum et des zones d'amplification importantes se développent.

L'objectif est maintenant de trouver une valeur de $K$ qui réduise les vibrations transmises à partir de $4000 \mathrm{~Hz}$ (et notamment la résonance à $4600 \mathrm{~Hz}$ ) et qui reste la plus élevée possible, car ce joint a aussi une fonction d'étanchéité qui demande une certaine transmission d'effort d'appui. En quelque sorte, on cherche l'assouplissement juste nécessaire permettant de filtrer au mieux les vibrations transmises par l'injecteur à la culasse. 


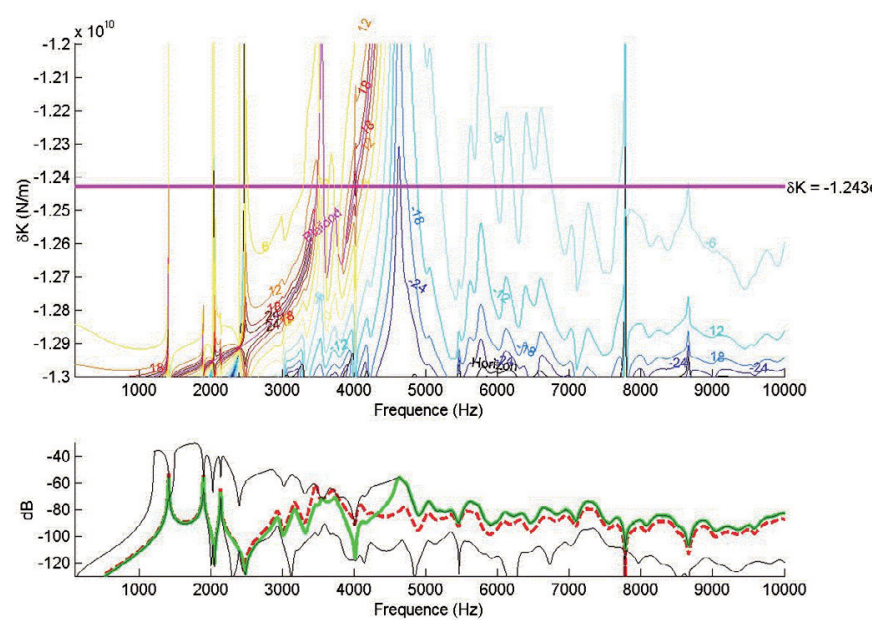

Fig. 5. Vue de l'interface graphique permettant le prédimensionnement en temps réel. La partie supérieure est un tracé de lignes iso-gains (en $\mathrm{dB}$ ) dans un espace fréquenceraideur. L'intersection d'une courbe représentant la raideur dynamique du joint (ici une droite horizontale) avec les lignes d'iso-gains permet d'estimer les variations de la réponse au point d'observation. Un tracé de cette dernière vient compléter l'interface.

Comme on l'a souligné dans la partie 1, mesurer la qualité de la modification ainsi réalisée ne se résume pas en termes de décibels gagnés à $4600 \mathrm{~Hz}$. En pratique, on va ici d'une part regarder l'atténuation obtenue sur tout un voisinage de cette résonance et d'autre part rester attentif à l'ensemble de la bande de fréquences pour contrôler les effets de la modification.

Ce problème d'optimisation complexe est résolu à l'aide des expressions et outils décrits à la section 1.3.3. Le tracé des lignes iso de gains se trouve sur la figure 5 . On remarque que les amplifications maximum forment approximativement sur ce diagramme une courbe croissante (entre 1 et $4 \mathrm{kHz}$ environ) traduisant l'évolution de la position du mode de suspension de l'injecteur en fonction de la variation de raideur.

Dans le cas traité ici, on trouve qu'une raideur $K_{1}=$ $5,7 \times 10^{8} \mathrm{~N} \cdot \mathrm{m}^{-1}$ permet de réduire significativement le niveau de la résonance à $4600 \mathrm{~Hz}$ et dans son voisinage proche, en profitant d'un creux de la courbe enveloppe supérieure (plafond) située à $4000 \mathrm{~Hz}$. L'assouplissement introduit a en fait permis de décaler le mode de $4600 \mathrm{~Hz}$ (mode de suspension de l'injecteur) à $4000 \mathrm{~Hz}$ où un nœud de vibration apparaît au point d'observation.

\subsubsection{Paramètre d'amortissement}

Une fois la valeur de raideur choisie, on effectue un pré-dimensionnement pour l'amortissement. Une démarche identique est pratiquée, en changeant le paramètre de raccordement $K$ qui est remplacé par $\eta$, amortissement hystérétique du joint anti-vibratoire, et en changeant la situation initiale qui comprend désormais un joint élastique de raideur $K_{1}$ identifiée précédemment.

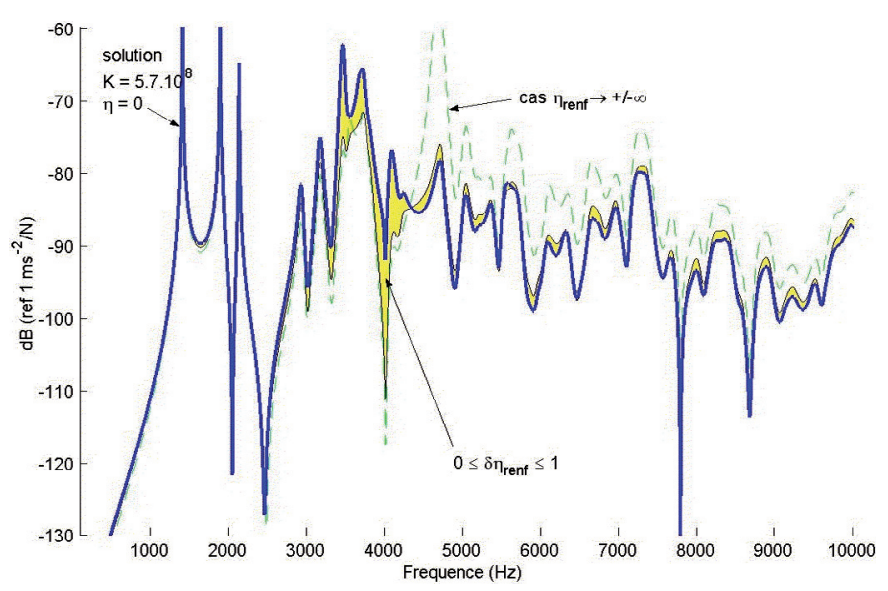

Fig. 6. Courbes enveloppes calculées pour le paramètre d'amortissement $\eta$ compris entre 0 et 1 ; en trait épais le cas sans amortissement (raideur $K 1=5,7 \times 10^{8} \mathrm{~N} . \mathrm{m}^{-1}$ ) ; en pointillé, la courbe asymptote pour $\eta \rightarrow \pm \infty$.

La figure 6 présente les courbes enveloppes obtenues pour une variation de $\eta$ comprise entre 0 et 1 . Il en ressort que l'amortissement du joint aura une influence importante pour la bande de fréquences comprises entre environ 3000 et $5000 \mathrm{~Hz}$. On notera également qu'un amortissement infini (trait vert pointillé) aurait naturellement des conséquences catastrophiques sur les objectifs de filtrages que nous nous sommes donnés.

En utilisant la même technique de dimensionnement que précédemment, on trouve qu'une valeur réaliste et efficace d'amortissement est environ $\eta_{1}=0,3$ pour les fréquences supérieures à $3 \mathrm{kHz}$. Le paramètre est peu influent en deça.

\subsubsection{Sensibilités}

Il est ensuite possible, toujours avec les mêmes méthodes, de quantifier la sensibilité aux deux paramètres $K$ et $\eta$ du joint anti-vibratoire pré-dimensionné précédemment. Il faut pour cela considérer l'état initial correspondant aux valeurs $K=K_{1}$ et $\eta=\eta_{1}$ et prendre la raideur comme nouveau paramètre de raccordement, variant par exemple entre $-2 \times 10^{8}$ et $2 \times 10^{8} \mathrm{~N}^{-m^{-1}}$. La figure 7 présente les effets de ces variations de raideur : cette plage de variation conduit globalement à des écarts d'au maximum $12 \mathrm{~dB}$, dans la zone la plus sensible située au voisinage de $4300 \mathrm{~Hz}$ sans pour autant faire (ré-)émerger de résonance marquée.

Ces informations de sensibilités sont importantes à ce stade de l'étude dans la mesure où la réalisation d'un tel joint présente des incertitudes importantes principalement liées à la nature des matériaux envisageables et leur évolutions dans le temps.

\section{Conclusion}

La méthode originale exposée ici permet un dimensionnement rapide mais non moins précis de raccords 


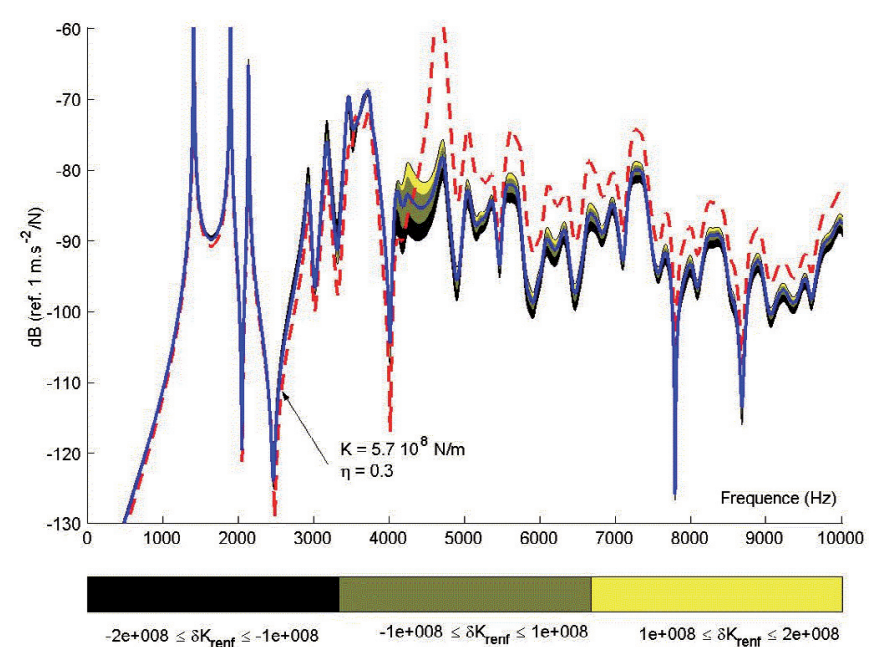

Fig. 7. Sensibilité à la raideur pré-dimensionnée. Enveloppes pour différentes plages de variation de la raideur spécifiée $\left(K_{1}=5,7 \times 10^{8} \mathrm{~N} \cdot \mathrm{m}^{-1}\right.$ et $\eta_{1}=0,3$ courbe en trait continu épais). La courbe en pointillé correspond à la situation de départ (joint en cuivre).

venant modifier une structure complexe. Les données d'entrée représentant les différents systèmes en jeu peuvent résulter de mesures directes, ou de modélisations détaillées en éléments-finis, ou encore de modèles réduits, de modèles hybrides, etc. Nous en avons donné ici un exemple d'application sur une structure moteur à laquelle on vient ajouter un joint filtrant avec amortissement.

La limitation principale (apparente) de ces méthodes porte sur le caractère localisé des modifications possibles. En réalité, le champ des applications n'en demeure pas moins très étendu dès lors qu'un travail d'identification soigné du degré de liberté de raccord est effectué, ce dernier étant à comprendre dans un sens généralisé. Ainsi, dans des modélisations éléments-finis, cela passe par la réalisation de « réductions » intelligentes : par des méthodes de condensation dynamique, par l'emploi de moyennes à partir des degrés de liberté de calcul, voire de rigidification de la zone de couplage (emploi d'éléments de type RBE2 ou RBE3 de NASTRAN, typiquement).

Des systèmes à connections multiples (plus de deux zones de raccordement) peuvent également être appréhendés en adoptant une approche pas à pas analogue à celle proposée dans cet article concernant le couple de paramètre raideur-amortissement.

Un autre avantage essentiel de la méthode se situe dans l'élaboration de critères et représentations utiles à l'ingénieur et simples à mettre en œuvre puisque basées sur des formulations analytiques. La gestion d'incertitudes sur les paramètres et résultats (la notion de ou), y compris l'amortissement, permet enfin de répondre à une préoccupation très actuelle dans le domaine du contrôle vibro-acoustique.

Les perspectives d'évolution des outils introduits ici sont nombreuses : allant de la construction d'outils spécialisés à l'insertion dans des boucles automatisées d'optimisation de structure.

\section{Références}

[1] A.H. Vincent, A note on the properties of the variation of structural res-ponse with respect to a single structural parameter when plotted in the complex plane, Technical report, Westland Helicopters Ltd., September 1973

[2] D.J. Ewins, Modal testing, theory, practice and application, Research Studies Press Ltd., $2^{\mathrm{e}}$ edition, 2000

[3] J.E. Mottershead, Structural modification for the assignment of zeros using measured receptances, J. Appl. Mech. 68 (2001) 791-798

[4] J.E. Mottershead, A. Kyprianou, H. Ouyang, Structural modification, Part 1: rotational receptances, J. Sound Vib. 284 (2005) 249-265

[5] A. Kyprianou, J.E. Mottershead, H. Ouyang, Structural modifica-tion, Part 2: assigment of natural frequencies and antiresonances by an added beam, J. Sound Vib. 284 (2005) 267-281

[6] M. Corus, E. Balmes, L. Billet, Amélioration de méthodes de modification structurale - application, Mécanique \& Industries 7 (2006) 139-145

[7] G.T.S. Done, A.D. Hughes, The response of a vibrating structure as a function of structural parameters, J. Sound Vib. 38 (1974) 255-266

[8] H.W. Hanson, N.J. Calapodas, Evaluation of the practical aspects of vibration reduction using structural optimization techniques, J. Am. Helicopter Soc. 25 (1979) $37-45$

[9] J.M. Lagache, Théorie et pratique du raccordement, Technical report, PSA Peugeot Citroën, 2004

[10] M.G. Tehrani, W. Wang, C. Maresb, J.E. Mottershead, The generalized Vincent circle in vibration suppression, J. Sound Vib. 292 (2006) 661-675

[11] H. Schwerdtfeger, Geometry of Complex Numbers, Circle geometry, Moebius Transformation, NonEuclidean Geometry, Dover Publications Inc., New York, $2^{\mathrm{e}}$ edition, 1979

[12] O. Sauvage, P. Burry, C. Perronnet, J.-M. Eickhoff, J.-M. Auger, Device for damping vibrations and combustion engine fitted with such a device, Application Number: EP20060300009, 2006 